\title{
Experimental observation of photonic bandgaps in hyperuniform disordered material
}

\author{
Weining Man, ${ }^{1}$ Marian Florescu, ${ }^{2}$ Kazue Matsuyama, ${ }^{1}$ Polin Yadak, ${ }^{1}$ Salvatore Torquato, ${ }^{4}$ Paul Steinhardt, ${ }^{2,3}$ \\ and Paul Chaikin ${ }^{4}$ \\ ${ }^{1}$ Department of Physics and Astronomy, San Francisco State University, San Francisco, CA 94132 \\ ${ }^{2}$ Department of Physics, Princeton University, Princeton, NJ 08544 \\ ${ }^{3}$ Princeton Center for Theoretical Science, Princeton University, Princeton, NJ 08544 \\ ${ }^{4}$ Department of Physics, New York University, New York, NY 20012 \\ weining@sfsu.edu
}

Abstract: We report the first experimental demonstration of photonic bandgaps (PBGs) in 2D hyperuniform disordered materials and show that is possible to obtain isotropic, disordered, photonic materials of arbitrary size with complete PBGs.

(C)2010 Optical Society of America

OCIS codes: $160.5293,160.5298$

Photonic bandgap materials have been studied extensively since their introduction in 1987. With materials having sizeable photonic bandgaps, an enormous range of technological developments in telecommunication industry, laser engineering, optical computing, spectroscopy, radiation and so on, would become possible, when light with selected frequencies can be directed along chosen paths or be confined within a specific volume [1]. To date, most photonic bandgap materials are periodic or quasiperiodic. Periodic materials can only have a restricted set of rotational symmetries, as dictated by the laws of crystallography, and so their structure and photonic bands are anisotropic. As a result, arranging an overlap of stopgaps to form a complete photonic bandgap can be difficult, typically requiring a combination of constituents with a large ratio of dielectric constants. [2,3]. Also, periodicity constrains the defect properties. These restrictions are disadvantageous for some applications.

In this paper, we report the first experimental demonstrations of photonic bandgaps in 2D hyperuniform disordered materials and show that is possible to obtain isotropic photonic materials of arbitrary size with complete PBGs (all polarizations \& directions). Our theoretical analysis finds good agreement with experimental data.

PBGs are commonly associated with long-range translational order and Bragg scattering, so the PBGs in disordered dielectric materials without long-range order are counterintuitive. In a recent theoretical paper, some of us presented an explicit approach for designing two-dimensional isotropic and disordered photonic bandgap materials [4]. It was argued that hyperuniformity, combined with uniform local topology and short-range geometric order can explain the origin of photonic band gaps in these disordered materials [4].

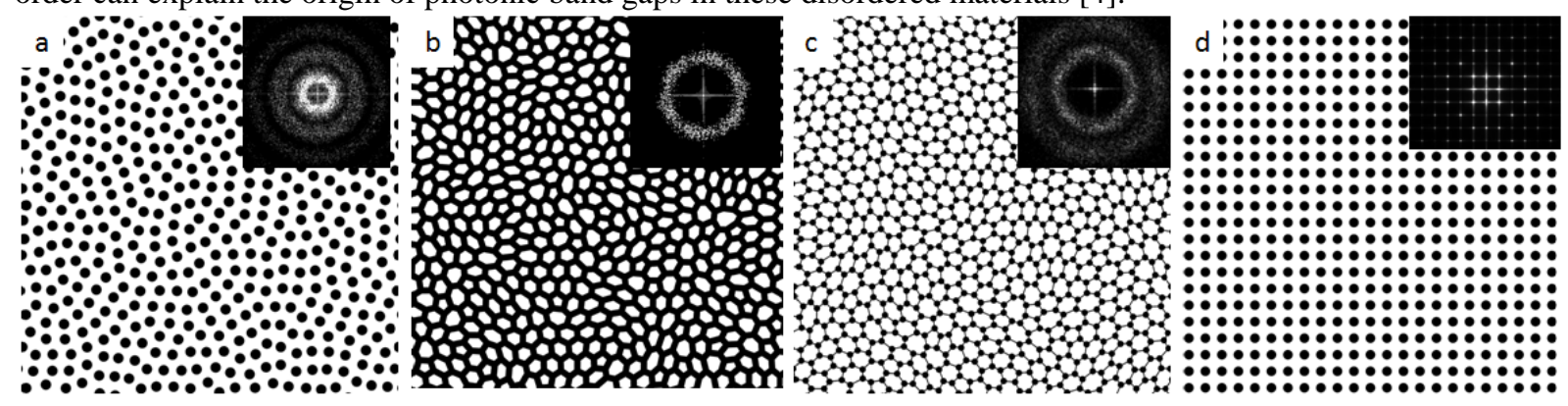

Fig. 1. (a) A Hyperuniform pattern decorated with identical dielectric $(\varepsilon=2.56)$ cylinders of radius $r$ processing a TM photonic bandgap. (b) A Hyperuniform pattern connected with dielectric $(\varepsilon=2.56)$ walls of uniform width $w$ processing a TE photonic bandgap. (c) A Hyperuniform pattern connected with dielectric $(\varepsilon=11.56)$ cylinders and walls processing a complete photonic bandgap $(\delta \mathrm{f} / \mathrm{f}=10 \%)$ in all directions and polarizations. (d) A square lattice decorated with identical dielectric $(\varepsilon=2.56)$ cylinders with similar filling fraction as the structure shown in Fig.1a. (Inserts: their structure factor $S(\boldsymbol{k})$, respectively).

A point pattern in 2D is hyperuniform, if the number variance $\sigma^{2}(R) \equiv\left\langle N_{R}^{2}\right\rangle-\left\langle N_{R}\right\rangle^{2}$ within a spherical sampling window of radius $R$ (in 2-dimension) grows more slowly than $R^{2}$. Hyperuniform patterns include all crystals, quasicrystals and a subset of disordered structures. In reciprocal space, hyperuniformity corresponds to the condition that the structure factor $S(\boldsymbol{k})$ approaches zero as the wavevector $|\boldsymbol{k}|$ approaches zero, (omitting forward scattering), i.e., infinite wavelength density fluctuations vanish. Hyperuniform patterns with a continuous, isotropic structure factor $S(\boldsymbol{k})$ that is precisely equal to zero for a finite range $\left(\boldsymbol{k}<\boldsymbol{k}_{\boldsymbol{c}}\right.$ for some finite wavenumber $\mathrm{k}_{\mathrm{c}}$ ) are called stealthy hyperuniform patterns, and their stealthiness can be parameterized by $\boldsymbol{k}_{\boldsymbol{c}}$. Fig.1 shows three hyperuniform patterns composed of cylinders, walls, or both designed to have a 2D TM, TE, or complete photonic bandgap respectively, together with a square lattice pattern for comparison. Hyperuniformity (a measure of the large scale 
fluctuations in the scattering center distribution) and short-range geometric order (e.g., small variation in the distance between vertices) combined with uniform local topology (all scattering centers have the same coordination number) strengthen the resonant Mie scattering [5] and can lead to the formation of complete PBGs [4].

We used stereolithography to fabricate these structures on $\mathrm{mm}$ to $\mathrm{cm}$ scales and used the corresponding microwave radiation to study their photonic properties. To measure the transmission photonic bandgaps, the experimental setup is similar to the one described in Ref. [2]. We first focused on fabricating materials designed to have optimal bandgaps for TM polarization. Each heterostructure is composed of air and dielectric material with $\varepsilon=2.56$. The samples were rotated along an axis perpendicular to the $2 \mathrm{D}$ patterned plane, and record the transmission was recorded every two degrees between 15 to $35 \mathrm{GHz}$.

For the disordered isotropic hyperuniform structure shown in Fig. 1a, we plot the calculated and measured TM transmission at normal incident (0 degree) in Fig. 2a. A clear transmission gap (about 4-5 GHz wide) centered at $22 \mathrm{GHz}$ can be observed. For the periodic square lattice structure shown in Fig 1d, we plot the calculated and measured TM polarization transmission at normal incident (X direction) and (45 degree $\mathrm{M}$ direction) in Fig. 2b and Fig. 2c, respectively. The experimental and theoretical results agree well.
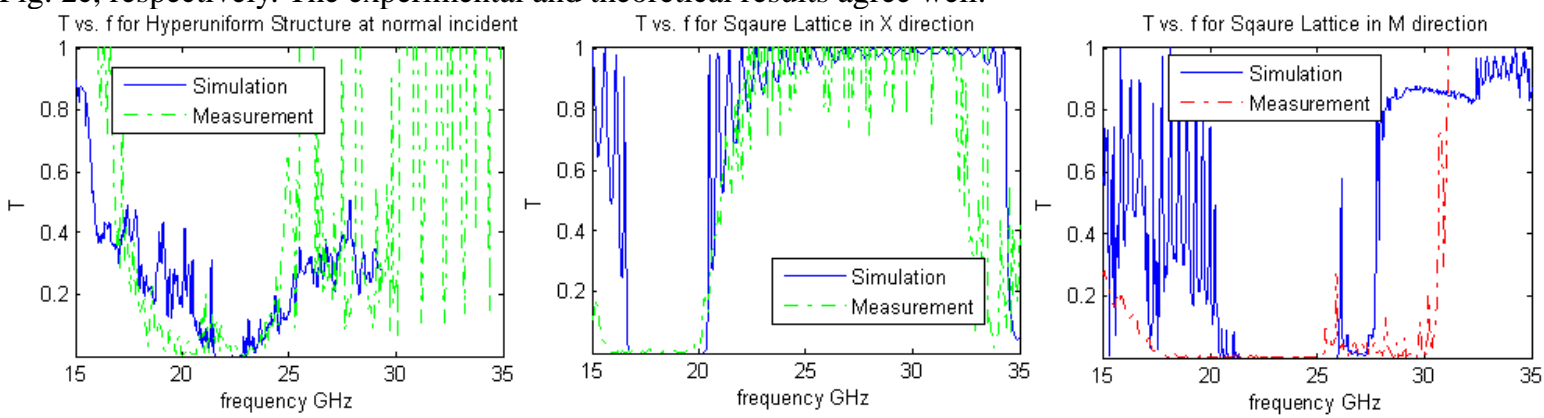

Fig. 2. (a) TM polarization transmission spectrum at normal incident for the hyperuniform structure shown in Fig.1a. (b)\&(c) TM polarization transmission spectrum at normal incident ( $\mathrm{X}$ direction) and at 45 degree ( $\mathrm{M}$ direction) for the square lattice structure shown in Fig. 1d.

For the square lattice structure, the stopgaps in the $\mathrm{X}$ and $\mathrm{M}$ directions are centered at different frequencies. As noted above, the anisotropy makes it hard to overlap stopgaps in all directions to form a complete PBG. By contrast, the stopgaps for the disordered hyperuniform structure are isotropic, as shown in Fig 3a. In Fig. 3 we use polar plots of $T(r=f, \theta=\theta)$ to present the measured Transmission (color) as a function of frequencies $(r=f)$ and angles $(\theta=\theta)$ [2]. The results for the hyperuniform structure show a complete TM transmission bandgap (circular ring of blue in Fig. 3a) deeper than $-15 \mathrm{~dB}$ from 18 to $23 \mathrm{GHz}$, while the largest bandgap (circular ring passing through the stopgaps) for the square lattice is much narrower.

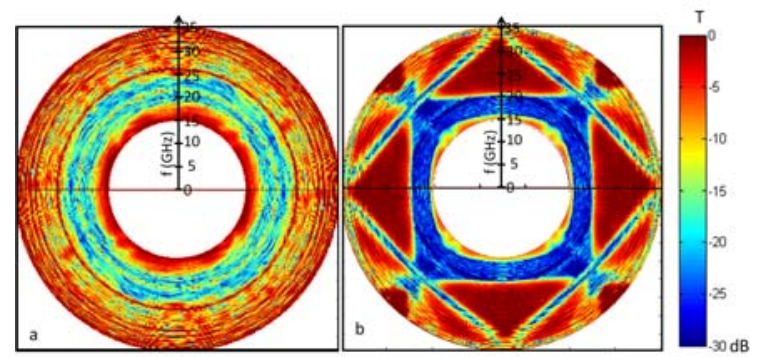

Fig. 3. (a) Measured transmission as a function of frequency and angle for the hyperuniform structure shown in Fig 1a. (b) Measured transmission as a function of frequency and angle for the square lattice structure shown in Fig $1 \mathrm{~d}$.

In summary, we have experimentally demonstrated TM photonic band gaps in 2D disordered hyperuniform dielectric material, at dielectric contrast as low as $\varepsilon=2.56$. The experimental results agree well with the theoretical predictions. Our next step will be to produce analogous hyperuniform disordered structures, which the theory predicts to have large TE PBGs and complete PBGs [4]. Disordered hyperuniform structures with substantial complete PBGs offer advantages in many applications, including optical insulator platform for planar optical circuits and isotropic thermal radiation sources and waveguides with arbitrary bending angles.

This work was supported by the Research Cooperation and the NSF Grant No. DMR-0606415. (MF, PJS, ST)

[1] John D. Joannopoulos, etc. Photonic Crystals: Molding the Flow of Light (Princeton University Press, Princeton, 2008)

[2] Weining Man, etc. Nature 436, 993-996 (2005).

[3] Marian Florescu, etc. Physical Review B 80, 155112 (2009)

[4] Marian Florescu, etc. PNAS, (in press, published online at http://www.pnas.org/content/ early/2009/11/12/0907744106).

[5] E. Lidorikis, etc. Phys. Rev. Lett. 81, 1405 - 1408 (1998) 